\title{
Optical Flow From A Least-Trimmed Squares Based Adaptive Approach
}

\author{
Ming Ye and Robert M. Haralick \\ Department of Electrical Engineering \\ University of Washington \\ Seattle, WA, 98195
}

\begin{abstract}
Optical flow estimation can be formulated as two regression stages, derivative estimation and optical flow constraints (OFC) solving. Traditional approaches use LeastSquares at both stages and are sensitive to assumption violations. To improve estimation accuracy especially near motion boundaries, we use a Least Trimmed Squares (LTS) estimator to solve the OFC, obtaining a confidence measure for each estimate; and at place with low confidence, we use another LTS estimator to robustify derivative estimation. This adaptive two-stage robust scheme has significantly higher accuracy than non-robust algorithms and those only using robust methods at the OFC stage. Advantages are illustrated on both synthetic and real data.
\end{abstract}

\section{Introduction}

Gradient-based optical flow estimation is generally composed of derivative estimation and optical flow constraints (OFC) solving two steps. Both steps involve optimization by pooling information in a certain neighborhood and are regression procedures in nature.

Classical approaches solve both regression problems in a Least-Squares (LS) sense. At places where the motion is multi-model, their results can be arbitrarily bad. To cope with this problem, a few robust regression tools such as MEstimators [3] [5] and Least Median of Squares (LMedS) estimators [6] [1] have been introduced to the OFC stage. However, choice of these tools usually lacks solid statistical justification and what technique is most appropriate remains an open problem. From conclusions on modern regression methods [10] [7] [8] [9] and characteristics of the optical flow constraints, we find the Least Trimmed Squares (LTS) technique to be more appropriate for the OFC step.

Meanwhile, as a very similar information pooling step, derivative calculation has seldom received proper attention in optical flow estimation. Crude derivative estimators are widely used. As the consequence, robust OFC (one-stage robust) methods still break down near motion boundaries. We calculate derivatives from an explicit 3D facet model [11]. This approach reveals that the derivative estimation step is also a regression problem, which can be robustified when the LS technique fails. We choose an LTS estimator for robust facet model fitting. Preliminary experimental results show that the two-stage LTS scheme permits correct flow recovery even at immediate motion boundaries.

We calculate a confidence measure for each estimate from the LTS OFC step, and update the derivatives and the flow vector if the measure takes a small value. By this means the one-stage and two-stage robust methods are carried out adaptively.

\section{Two-Stage Linear Regression Model}

The linear regression model assumes that the relationship between the observation vector $Y$ and the unknown parameter $\Theta$ is

$$
X^{N \times M} \Theta^{M \times 1}+\xi^{N \times 1}=Y^{N \times 1},
$$

where $X$ is the design matrix and $\xi$ is the error vector. $\Theta$ is estimated as $\hat{\Theta}=\operatorname{argmin}_{\Theta} F(r)$, where $r$ is the residual error $Y-\hat{Y}=Y-X \hat{\Theta}$. The criterion function $F(r)$ differs among estimators depending on what error model is assumed. The LS estimator assumes $\xi$ to be additive iid Gaussian with zero mean and variance $\sigma^{2}$ and thus $F(r)=$ $\|r\|^{2}=\sum_{i=1}^{N} r_{i}^{2}$.

Optical Flow Constraint Following [4] we constrain the optical flow vector $V=(u, v)^{\prime}$ at $(x, y, t)$ by $A V+\xi=b$ where

$$
A=\left(\begin{array}{cc}
I_{x} & I_{y} \\
I_{x x} & I_{x y} \\
I_{y x} & I_{y y} \\
I_{t x} & I_{t y}
\end{array}\right) \quad b=-\left(\begin{array}{c}
I_{t} \\
I_{x t} \\
I_{y t} \\
I_{t t}
\end{array}\right)
$$

We further assume that flow vectors in each small neighborhood of $N_{s}$ pixels is constant, so each vector $V$ conforms to $N_{s}$ such sets of constraints simultaneously. This forms our 
optical flow constraint [11], a linear regression model

$$
A_{s} V+\xi=b_{s}
$$

where $A_{s}=\left(A_{1}^{\prime}, A_{2}^{\prime}, \ldots, A_{N_{s}}^{\prime}\right)^{\prime}, b_{s}=\left(b_{1}^{\prime}, b_{2}^{\prime}, \ldots, b_{N_{s}}^{\prime}\right)$, and $A_{i}, b_{i}$ are defined as above. This constraint uses both first and second order derivatives. When $N_{s} \geq 2$, an alternative is to use the first order constraints only [1] [2].

Derivative Estimation Derivatives need to be estimated before solving the OFC. Most popular derivative estimators [2] are neighborhood masks, which essentially come from facet models. The facet model basically characterizes each small image data neighborhood by a signal model and a noise model so that operations can be done on the signal model and the error can be analyzed quantitatively. We use a 3D cubic polynomial on a $N_{t} \times N_{r} \times N_{c}$ image support as the signal model and estimate the polynomial coefficient vector $a$ from the linear regression model

$$
D a+\xi=J
$$

where $J$ is the observed image data vector and $D$ is the design matrix composed of polynomial bases. Once $a$ is found, the derivatives are merely scaled versions of its elements [11].

So far most optical flow estimators solve both regression problems using LS estimators. However as it is well-known, an LS estimator might fail at the presence of a single gross error either in $Y$ (y-outlier) and or in $X$ (leverage point) (Eq.(1)), i.e., it has a breakdown point of $0 \%$ [7]. Since in optical flow estimation gross errors happen frequently due to brightness variation, motion boundaries and so on, we need to relax LS's error model and use more robust estimators.

\section{LTS Optical Flow Constraint}

We observe that (i) both y-outliers and leverage points might happen in Eq.(2) because both $A_{s}$ and $b_{s}$ are composed of derivative estimates; (ii) leverage points are roughly twice as much as y-outliers; (iii) a significant portion of the constraints might be gross errors, when, for example, multiple motion models happen in a neighborhood; and (iv) the number of constraints are relatively small. Therefore the desired estimator to solve the OFC should be resistant to both types of gross errors, have a high breakdown point and good statistical efficiency on a small sample size.

M-estimators [3][5] and LMedS estimators [6][1] were previously used at the OFC-stage. M-estimators are resistant to y-outliers and have relatively high statistical efficiency, but they have a low breakpoint of about $1 / M$ (Eq.(1)) and are vulnerable to leverage points [7]. The
LMedS estimator [7] is resistant to both types of gross errors and has a high breakdown point of $50 \%$, but it has extremely low statistical efficiency, which means it tends to perform poorly when there is no gross errors.

The Least Trimmed Squares estimator [7] was introduced to repair the low efficiency of LMedS. It is defined as

$$
\hat{\Theta}=\operatorname{argmin}_{\Theta} \sum_{i=1}^{h}\left(r^{2}\right)_{i: n}
$$

where $\left(r^{2}\right)_{1: n} \leq \ldots \leq\left(r^{2}\right)_{n: n}$ are the ordered squared residuals. By excluding the largest squared residuals from the LS criterion function, LTS produces a fit insensitive to the gross errors. Owning almost all merits of LMedS and better statistical efficiency, LTS is usually preferred to LMedS [10] [8] [9].

\section{LS or LTS: Adaptive Derivative Estimation}

By default we solve the 3D cubic facet model in an LS sense to find the derivatives. When the estimation quality is poor, we update the derivatives from robust facet model fitting. To reduce sensitivity to noise and computation we use a 3D quadratic facet model for this purpose. As the facet parameter $a$ size is large (10), and the breakdown point has to be high, the LTS estimator is a better choice than M- and LMedS estimtors. Note that it is not appropriate to apply LTS facet model fitting uniformly, because LTS tends to have lower statistical efficiency than LS when there is no gross error, and it involves much more computation. Therefore the LTS facet model should be used when and only when the estimation fails due to the LS facet quality. We take the coefficient of determination $\left(R^{2}\right)$ [7] from the LTS OFC step as the confidence measure of the flow estimate, and detect LS facet failures by examining if $R^{2}<T$. $R^{2} \in[0,1]$ measures the proportaion of observation variability explained by the regression model.

It is worth mentioning that our and Bab-Hadiashar's OFC stages use a similar local optimization formulation, with the difference that he uses LMedS while we use LTS as the regression tool. Both of us detect estimates with low $R^{2}$ values but we treat them very differently. He merely declares them as unreliable, whereas we apply a two-stage LTS to improve their accuracy.

\section{LTS Implementation Based on FAST-LTS}

To solve a constraint of $n$ equations and $p$ parameters, the brute force LTS implementation, for each of the $C_{n}^{p}$ equation subsets, computes $\hat{p}$ and the fitting error vector $r^{2}$, sorts $r^{2}$ and finds the LTS error, and finally takes the $\hat{p}$ with the smallest error as the solution. This is computationally prohibitive. In practice LTS is usually approximated by using a part of the equation subsets [7]. Different subset selection 


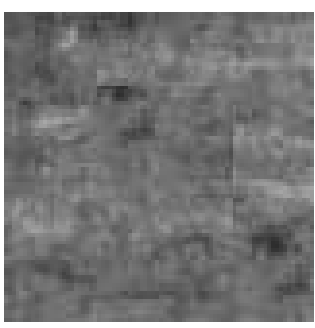

(a) Central frame $(64 \times 64)$

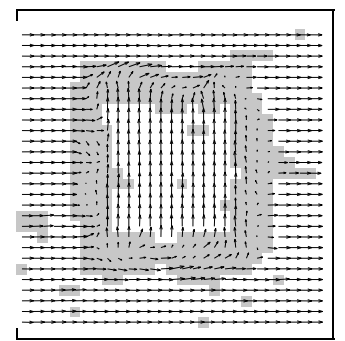

(e) LS-LS

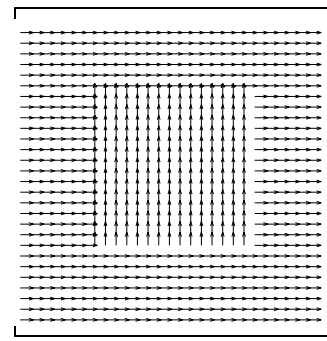

(b) Correct flow

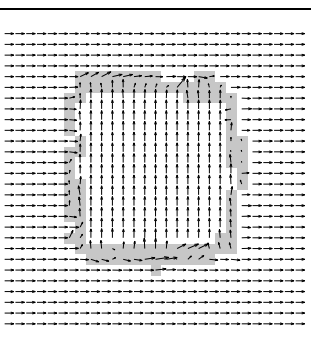

(f) LS-LTS

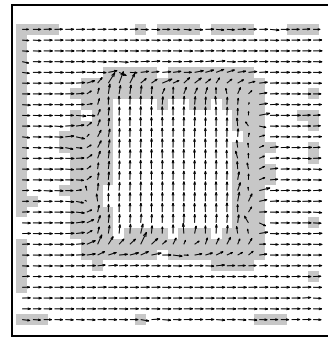

(c) Black

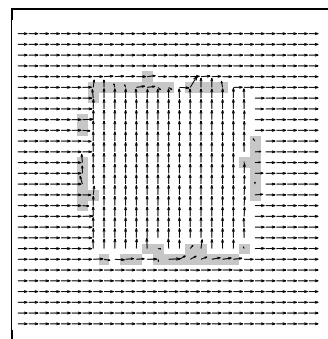

(g) LTS-LTS

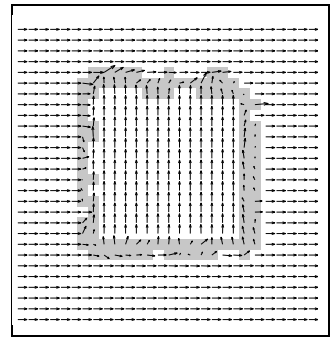

(d) Bab-Hadiashar

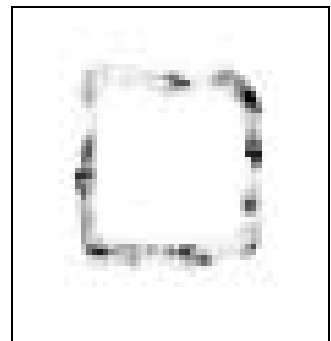

(h) Confidence measure $R^{2}, T=0.99$

Figure 1. Experiments on the synthetic data

schemes have different converging rates. Our implementation is based on FAST-LTS [9], one of the most efficient algorithms available.

\section{Experiments and Analysis}

We demonstrate on both synthetic and real data how optical flow accuracy significantly improves as the LSbased method (LS-LS), the one-stage robust method (LSLTS) and the two-stage robust method (LTS-LTS) are used. We also compare our results with those from Black and Anandan's dense regularization approach [3] and BabHadiashar's LMedS based approach [1]. The results were computed using their own $\mathrm{C}$ code, all parameters being default.

\subsection{Synthetic Sequence}

Fig. 1(a),1(b) are the central frame and the correct flow field of the synthetic sequence (velocity magnitude: 1 pixel/frame, image size: $64 \times 64)$. Both facet and OFC neighborhood sizes are set to 5 pixels for Bab-Hadiashar's and our algorithms. LS-LTS uses the first order OFC only.

We calculate the error percentage as the quantitative accuracy measure. It is the error vector magnitude normalized by the true velocity magnitude and multiplied by 100 . The average error percentages on the entire flow field (AEP) and the motion boundary of width 9 pixels (AEPB) are summarized in Tab.1. LTS-LTS is applied at places with $R^{2}<0.99$. The flow fields estimated from five algorithms are given in Fig.1. We shade estimates with error percentages larger than $0.1 \%$. All flow fields are subsampled by 2 .

\begin{tabular}{|l|rr|}
\hline Technique & AEP(\%) & AEPB $(\%)$ \\
\hline LS-LS & 18.83 & 50.61 \\
Black & 11.88 & 29.02 \\
Bab-Hadiashar & 8.03 & 26.24 \\
LS-LTS & 7.53 & 24.59 \\
LTS-LTS & 4.75 & 15.51 \\
\hline
\end{tabular}

\section{Table 1. Average error percentage compari-} son on the synthetic data

Following observations can be obtained from this experiment. All robust methods out-perform the pure LS method. Possibly due to poor derivative quality, and M-estimators' inherent problems of low breakdown point and leverage point sensitivity, Black's result looks more noisy. LTS is better than LMedS in the OFC stage. LTS derivative estimation significantly reduces boundary errors.

\subsection{The Pepsi Sequence}

This is a real image sequence in which a Pepsi can and background move approximately 0.8 and 0.35 pixels to the left respectively (Fig.2(a)). Black [3] used this sequence to demonstrate the motion boundary preserving capability of their algorithm. We show subsampled flow fields of four techniques in Fig. 3 and the (linearly scaled) horizontal flow values in Fig.2. 


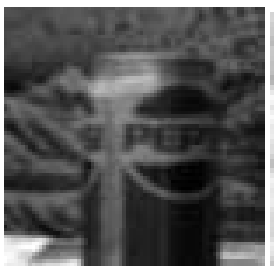

(a) Central frame, $64 \times 64$

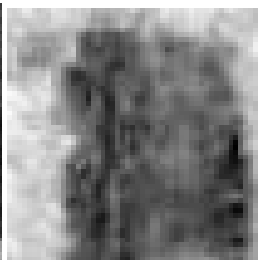

(b) Black

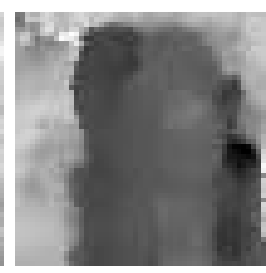

(c) Hadiashar

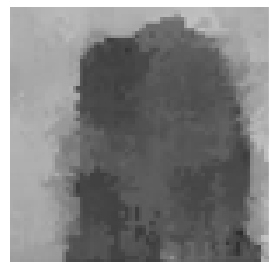

(d) LS-LTS

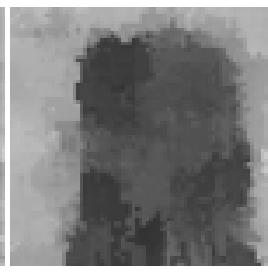

(e) LTS-LTS

\section{Figure 2. Pepsi: central frame and horizontal flow (darker pixels mean larger speeds to the left)}

Black's result (Fig. 3(a),2(b)) looks bumpy and smeared and does not have proper contrast of the foreground and background. Bab-Hadiashar's result (Fig. 3(b),2(c) has significant vertical speed components in the upper-left and the lower parts, and the flow is still over-smoothed. Fig. 3(c),2(d) is the result of LS-LTS (1st and 2nd order constraint). Motion contrast and discontinuities are much clearer. LTS-LTS (Fig. 3(d),2(e)) updated LS-LTS estimates with $R^{2}<0.75$ and significantly improved the boundary accuracy.

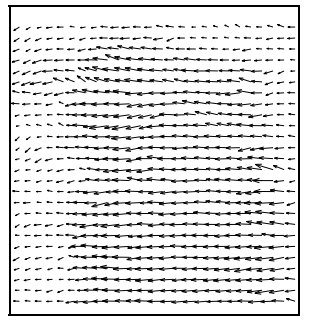

(a) Black

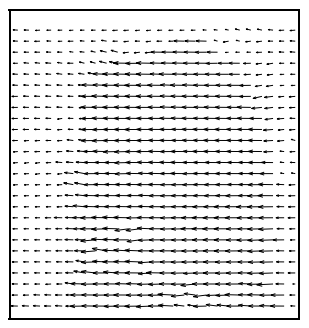

(c) LS-LTS

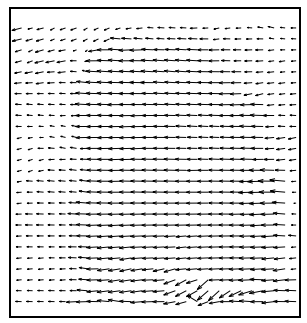

(b) Bab-Hadiashar

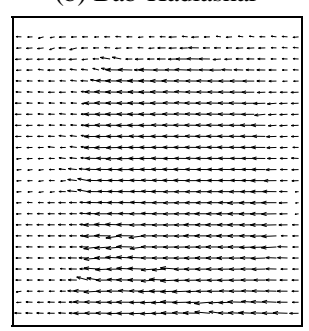

(d) LTS-LTS
Figure 3. Pepsi: estimated flow fields

\section{Conclusion and Discussion}

The primary contribution of this work that it formulates optical flow estimation as two regression problems and adaptively solves them using one-stage or two-stage LTS methods. It carefully analyzes the characteristics of the two regression stages and chooses robust tools correspondingly. Preliminary experimental results on both synthetic and real image sequences verified the effectiveness.

The two-stage robust method involves heavy computa- tion. A less expensive robust facet fitting method is being sought. The efficiency of $R^{2}$ as the one/two stage criterion needs validation. We are trying to develop a more reliable criterion with other measures such as outlier percentage combined. Although LTS is generally preferred to LMedS, their relative merits in this particular problem needs fuller investigation. The constant local motion model may readily extends to the affine model for higher accuracy. As this technique exhibits excellent boundary performance, we expect to find its application in motion segmentation.

Since derivative estimation is a fundamental step of many computer vision problems, and most optimization problems can be fit into the regression framework, conclusions of this paper may extend to other fields.

\section{References}

[1] A. Bab-Hadiashar and D. Suter, "Robust Optical Flow Estimation", IJCV, Vol. 29, No. 1, pp. 59-77, 1998

[2] J.L. Barron, S. S. Beauchemin and D. J. Fleet, "Performance of Optical Flow Techniques", IJCV, Vol. 12, No. 1, pp. 43-77, 1994

[3] M. J. Black and P. Anandan, "The Robust Estimation of Multiple Motions: Parametric and Piecewise-Smooth Flow Fields", CVIU, Vol. 63, No. 1, pp. 75-104, 1996

[4] R. M. Haralick and J. S. Lee, "The Facet Approach to Optic Flow", Proc. of IUW, pp. 84-93, 1983

[5] E. Memin and P. Perez, "Dense Estimation and Object-Based Segmentation of the Optical Flow with Robust Techniques", PAMI, Vol. 7, No. 5, pp. 703-19, 1998

[6] E. P. Ong and M. Spann, "Robust Optical Flow Computation Based on Least-median-of-Squares", IJCV, Vol. 31, No. 1, pp. 51-82, 1999

[7] P. J. Rousseeuw and A. M. Leroy, Robust Regression and Outlier Detection, John Wiley and Sons, 1987

[8] Rousseeuw, P.J. and Van Aelst, S., "Positive-Breakdown Robust Methods in Computer Vision", Computing Science and Statistics, Vol. 31, Interface Foundation of North America, Inc., pp. 451-460, 1999

[9] P. J. Rousseeuw and K. Van Driessen, "Computing LTS Regression for Large Data Sets", Tech. Report, Univ. of Antwerp, submitted.

[10] R. R. Wilcox, Introduction to Robust Estimation and Hypothesis Testing, Academic Press, 1997

[11] M. Ye and R. M. Haralick, "Image Flow Estimation Using Facet Model and Covariance Propagation", Vision Interface: Real World Applications of Computer Vision, pp. 209-241, World Scientific, 2000 